\title{
Étude et prise en compte de la variabilité spatiale
}

\section{A. BOLLE}

Université de Liège

(Belgique)

Chemin des Chevreuils $1-B-4000$ Liège
Après un bref rappel de la nature et de l'origine de la variabilité des caractéristiques géotechniques des sols et des massifs rocheux, le choix d'un modèle est proposé. La variabilité est représentée par l'évolution spatiale de la moyenne et par la dispersion autour de cette moyenne. Cette dispersion manifeste généralement une structure plus ou moins régulière en liaison avec la périodicité des phénomènes géologiques ou anthropiques, caractérisée par Yauto-corrélation.

Les effets de cette variabilité et de sa structure d'autocorrélation peuvent être analysés par des méthodes de calcul probabiliste adaptées, autorisant l'utilisation de grands nombres de variables aléatoires.

Quelques exemples d'application illustrent les propositions formulées.

Mots-clés : sols, variabilité spatiale, auto-corrélation, techniques de perturbation.

\section{Investigation and allowance for the spatial variability}

After a short recall about the nature and the origin of the variability of the geotechnical characteristics in soils and rock masses, a model is suggested. The variability is modelled by the spatial trend and by the scattering around that mean value. Generally the dispersion exhibits a more or less regular structure, related to the periodicity of the geological phenomena, characterised by the autocorrelation. The effects of that variability and of its autocorrelation structure can be analysed by sulted probabilistic computational methods, allowing to use large numbers of random variables. Some case studies are proposed as examples.

Koy words: soils, spatial variability, autocorrelation, perturbation techniques. 


\section{Introduction}

Le présent exposé veut s'adresser à des praticiens, et non à des mathématiciens. Dans un souci de clarté, de nombreuses simplifications parfois assez radicales ont été apportées aux hypothèses et aux développements mathématiques.

Les quelques notions de base servant à modéliser la variabilité spatiale des paramètres géotechniques des sols et des massifs rocheux sont brièvement rappelées ci-dessous,

Ainsi, notre approche de la variabilité spatiale supposera d'abord que les valeurs mesurées ne reflètent que la seule variabilité naturelle des propriétés du matériau, en négligeant les autres sources de dispersion, en particulier les erreurs de mesure.

Ensuite, notre échelle d'observation de la variabilité correspondra à celle qui concerne le constructeur. Elle sera comprise entre, d'une part, les dimensions de la couche de terrain considérée comme « homogène en grand » et, d'autre part, la dimension du volume d'échantillonnage de la grandeur mesurée. Les dimensions de ce volume d'échantillonnage peuvent aller de quelques centimètres pour des mesures en laboratoire, à plusieurs décimètres, voire plusieurs mètres, pour les techniques d'investigation in situ (pressiomètre, pénétromètre).

La modélisation utilisée repose sur un choix arbitraire, à savoir celui des champs aléatoires limités aux moments statistiques d'ordre deux. En termes simples, les fluctuations des paramètres dans l'espace (et éventuellement dans le temps) seront décrites par leurs moyennes, leurs variances et leur auto-corrélation.

Ce choix délibéré de la notion d'auto-corrélation permet de décrire par des modèles d'une écriture simple, comportant très peu de paramètres, divers types de grandeurs géotechniques mesurées sur terrain. II permet également, en profitant de certaines propriétés mathématiques de la notion de corrélation, de proposer des techniques de simulation simples et efficaces.

\section{2}

\section{Les champs aléatoires}

Il existe d'autres possibilités de modélisation, comme l'approche de Matheron (1965) basée sur le concept de fonctions aléatoires, qui se concrétise par la représentation du variogramme, mais elles ne seront pas développées ici. On utilisera exclusivement les champs aléatoires homogènes en se limitant à l'information statistique de second ordre, selon Vanmarcke et al. (1986).

Les grandeurs mesurées, par exemple la valeur prise par un paramètre géotechnique caractérisant le comportement d'une couche de sol, sont alors représentées par :

- une fonction décrivant l'évolution, sur le domaine considéré, des moyennes et variances locales, c'est-àdire estimées sur un petit sous-domaine;

- une fonction d'auto-corrélation, à une ou plusieurs dimensions.

La figure 1 présente l'exemple d'un paramètre géotechnique mesuré en fonction de la profondeur. La pre- mière composante du modèle va prendre en compte l'évolution moyenne avec la profondeur, ou dérive, qui peut être ici assimilée à une droite.

En soustrayant cette dérive, il est alors possible de ramener les valeurs mesurées à un champ aléatoire stationnaire, c'est-à-dire un ensemble de valeurs aléatoires dont la moyenne et la variance locales sont des constantes.

On obtient ainsi une nouvelle variable dite stationnaire, dont la moyenne est constante sur le domaine et souvent choisie égale à zéro. Cette variable sera ensuite caractérisée par sa variance, mesurant l'importance de la dispersion autour de la moyenne, et par la structure spatiale (ou éventuellement spatio-temporelle) de cette variance, décrite par l'auto-corrélation.

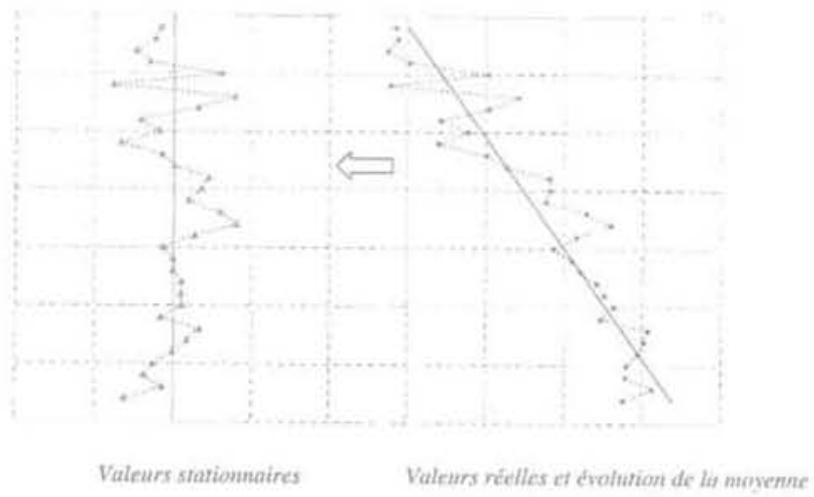

HG. Exemple de stationarisation de mesures géotechniques.

Example of stationarisation of geotechnical data.

\section{La fonction d'auto-corrélation}

\section{Définition}

La fonction d'auto-corrélation permet de décrire la structure de la dispersion qui subsiste après l'élimination de la dérive. On peut notamment observer des oscillations plus ou moins régulières liées à la périodicité des processus géologiques, combinées à des variations plus erratiques.

En se référant à la figure 1, on désignera par W(Z) la variable rendue stationnaire, fonction de la profondeur $Z$. Sa dispersion résiduelle est mesurée par $\sigma^{2}(W)$, la variance, supposée constante.

Sur le diagramme, on peut constater que deux mesures réalisées à des profondeurs voisines ont tendance à présenter de valeurs proches, tantôt inférieures, tantôt supérieures à la moyenne. Par contre, des mesures plus éloignées évoluent de manière plus indépendante. Ceci peut se traduire par la notion d'auto-corrélation, qui exprime la corrélation entre les valeurs $W(Z)$ observées et ces mêmes valeurs $W(Z)$ décalées d'une distance notée $\xi$.

La valeur de l'auto-corrélation est obtenue par une formule statistique similaire à celle utilisée pour esti- 
mer la corrélation entre deux grandeurs différentes. Elle a la forme d'un coefficient de corrélation :

$$
\begin{aligned}
& \rho(\xi)=\frac{\operatorname{cov}(W(Z), W(Z+\xi))}{\sigma^{2}(W)} \\
& \equiv \frac{1}{\sigma^{2}(W)}\left\{\frac{1}{N^{\prime}-1} \sum_{i=1}^{N}\left(W\left(Z_{i}\right) \times W\left(Z_{i}+\xi\right)\right)\right\}
\end{aligned}
$$

Si l'on fait varier la valeur du décalage $\xi$, on obtient différentes valeurs de la corrélation qui forment le diagramme expérimental d'auto-corrélation, ou auto-corrélogramme, tel celuî représenté sur la figure 2. Par définition, on a toujours $\rho(0)=1$.

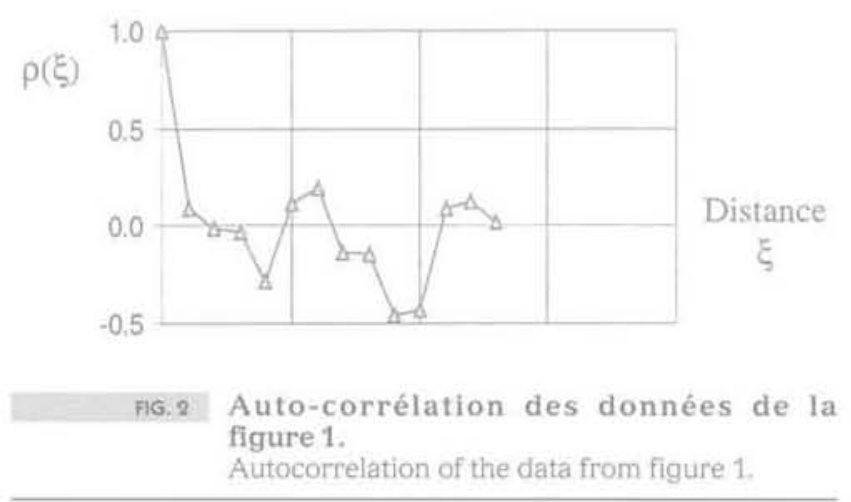

\section{Constatations experimentales}

Deux exemples d'auto-corrélogrammes, correspondant à des mesures réelles, vont illustrer certaines caractéristiques générales observées dans les sols et les massifs rocheux naturels.

À l'occasion d'un programme de recherche sur le comportement des pieux. De Beer et al. (1977) ont réalisé un grand nombre d'essais géotechniques sur un site comportant une épaisse couche d'argile assez homogène. La figure 3 est l'auto-corrélogrammme moyen des 20 essais de pénétration au cône réalisés à faible distance l'un de l'autre dans cette couche homogène, obtenu par Maertens (1990).

À l'occasion de l'étude d'une fondation de grand pont en arc dans un massif rocheux très perturbé, Bolle $(1988,1994)$ et Bolle et al. (1989) ont analysé la variabilité des 191 valeurs du module pressiométrique mesurées dans une série de forages verticaux et inclinés réalisés à l'emplacement d'une culée. Devant l'impossibilité de dégager une représentation stratigraphique cohérente, le massif rocheux a été assimilé à un corps isotrope très hétérogène, et son auto-corrélation, représentée sur la figure 4, a été étudiée sur le volume en utilisant une notion isotrope de la distance $\xi$.

En se basant sur ces deux exemples, on peut caractériser l'allure habituellement observée pour l'auto-corrélation de propriétés géotechniques, à savoir : une décroissance monotone à partir de l'origine, suivie par des oscillations plus ou moins régulières autour de zéro.

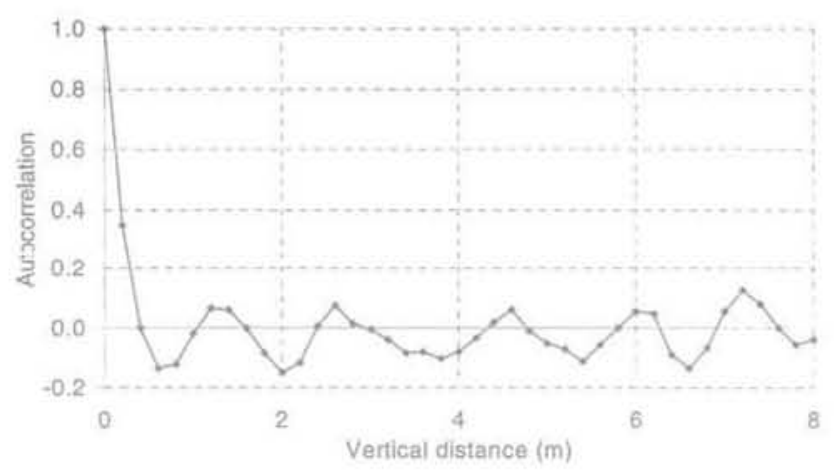

FiG.3 Auto-corrélogramme vertical (1D) moyen de 20 essais CPT.

Mean vertical autocorrelogram (1D) of 20 CPT tests.

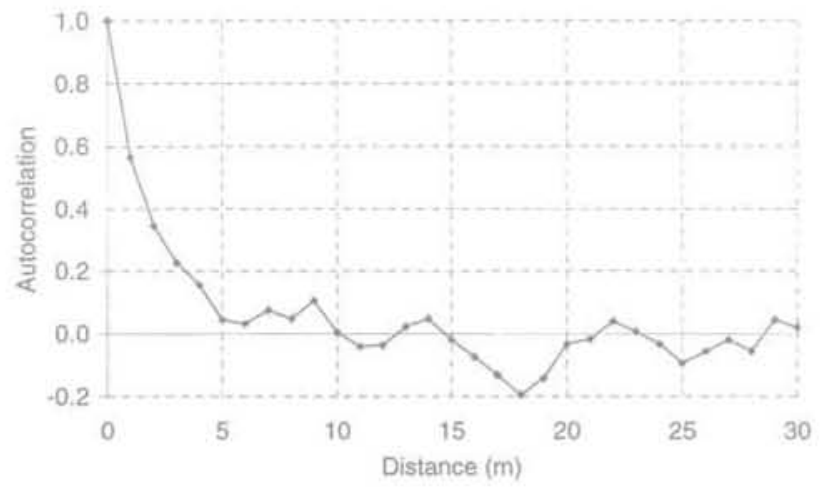

FiG. 4 Auto-corrélogramme isotrope 3D de 191 modules pressiométriques. Isotropic 3D autocorrelogram of 191 pressuremeter moduli.

\section{Critique des modèles classiques}

La plupart des modèles proposés dans la littérature pour représenter ces fonctions d'auto-corrélation expérimentales se contentent de représenter la décroissance au voisinage de l'origine. La fonction $p(\xi)$ passe ainsi de l'unité à zéro de manière monotone, puis reste nulle (Fig. 5). Les oscillations, toujours présentes de manière significative dans les auto-corrélogrammes expérimentaux, n'apparaissent pas dans ces modèles classiques.

Or, on peut montrer que ces oscillations sont une conséquence directe du caractère stratifié, lité, à motifs répétitifs, des formations naturelles, et qu'elles en constituent donc une composante essentielle. Un milieu

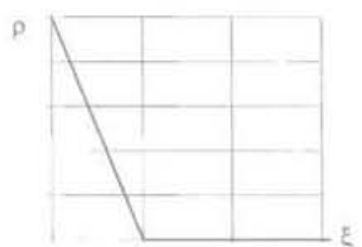

(a) linéaire

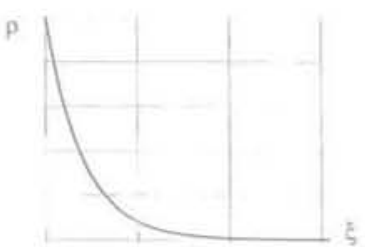

(b) exponenticl

Fig.5 Deux modèles classiques d'autocorrélation.

Two classical autocorrelation models. 
stratifié présente nécessairement ce comportement oscillant au-delà de la partie décroissante initiale.

Imaginons une couche de sol idéalisée formée d'une alternance régulière de deux matériaux similaires, mais de caractéristiques moyennes légèrement différentes (Fig. 6). On ajoute ensuite de la dispersion à ce modèle idéal (irrégularités dans l'alternance des sous-couches, variations erratiques et erreurs de mesure), de manière à se rapprocher d'une situation naturelle plausible. Dans une approche d'ingénieur, un tel sol sera généralement considéré comme une seule couche homogène « en grand $n$.

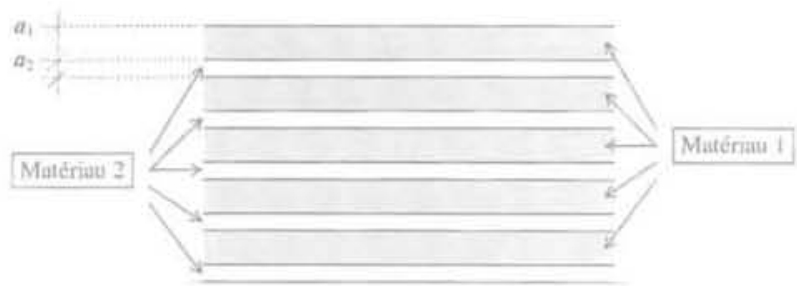

FIG.6 Modèle idéalisé de massif stratifié. Idealised model of a stratified soil mass.

On peut montrer (Bolle, 1990) que l'auto-corrélation d'un tel milieu ne dépend pas de la dispersion des caractéristiques, mais qu'elle est fonction uniquement de la géométrie de ce massif zoné. Les auto-corrélogrammes obtenus se rapprochent des courbes expérimentales habituellement observées (Fig. 7), avec notamment de larges oscillations assez régulières autour de zéro.

La modélisation de l'auto-corrélation ne peut donc pas, comme les modèles classiques évoqués plus haut, ignorer cet aspect. Le nombre de paramètres intervenant dans la définition de telles fonctions devra toutefois rester très limité, car la quantité d'information réellement significative à ce niveau de l'analyse est souvent très réduite.

On vient de voir que les paramètres de l'auto-corrélation sont de nature géométrique. En conséquence, outre les longueurs qui caractérisent les alternances internes, une autre longueur devrait de toute évidence jouer un ròle important, à savoir la dimension du volume d'échantillonnage de la grandeur mesurée.

\section{4}

\section{Le spectre de puissance}

\section{1}

\section{Définition}

Par une transformation du problème, familière en électricité et en traitement du signal, on va faire apparaître de manière explicite les divers paramètres géométriques et leur importance relative.

Vanmarcke et al. (1986) rappellent que la fonction d'auto-corrélation $\rho(\xi)$ peut être exprimée de manière équivalente sous la forme d'un spectre de puissance $s(\kappa)$, à l'aide de la paire de transformations de WienerKhintchine :

$$
\rho(\xi)=\int_{-\infty}^{-} s(\kappa) \cdot e^{i k \xi} \cdot d \kappa \Leftrightarrow s(\kappa)=\frac{1}{2 \cdot \pi} \int_{-\infty}^{\infty} \rho(\xi) \cdot e^{i k \xi} \cdot d \xi
$$

dans lesquelles la variable $\kappa$ a la dimension de l'inverse d'une longueur.

Ces fonctions sont paires en $\kappa$ et $\xi$, et la fonction de densité spectrale peut être définie de manière plus simple par $g(\kappa)=2 . s(\kappa)$. La transformation se réduit alors à une paire de transformées de Fourier en cosinus, avec les deux variables $\kappa$ et $\xi$ positives :

$$
\rho(\xi)=\int_{0}^{\infty} g(\kappa) \cdot \cos (\kappa \cdot \xi) \cdot d x \Leftrightarrow g(\kappa)=\frac{2}{\pi} \int_{0}^{\infty} \rho(\xi) \cdot \cos (\kappa \cdot \xi) \cdot d \xi(3)
$$

Les modèles classiques simples déjà illustrés plus haut, dans lesquels $\rho(\xi)$ reste positif, sont définis par un seul paramètre. La figure 8 présente côte à côte les auto-corrélogrammes et les spectres de puissance correspondants.

On remarque que, pour chacun de ces exemples, le spectre de puissance présente un maximum en $k=0$, suivi d'une décroissance. Compte tenu de la signification de la variable $\kappa$, inverse d'une longueur, ce maximum correspondrait ainsi à un phénomène de longueur (ou de portée) infinie, ce qui n'est guère compatible avec la réalité physique d'une couche de sol ou de roche, nécessairement de dimensions limitées.

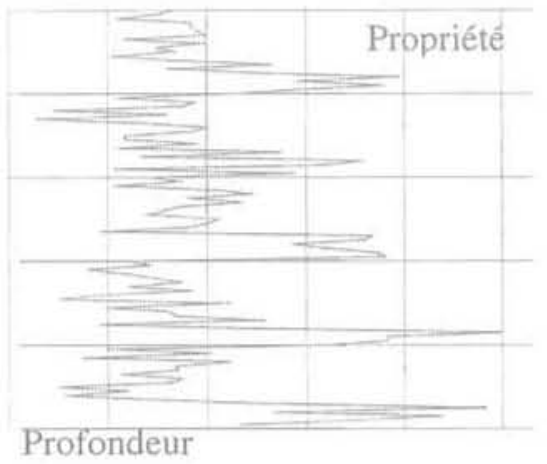

(a) Variation de la propriété avec la profondeur

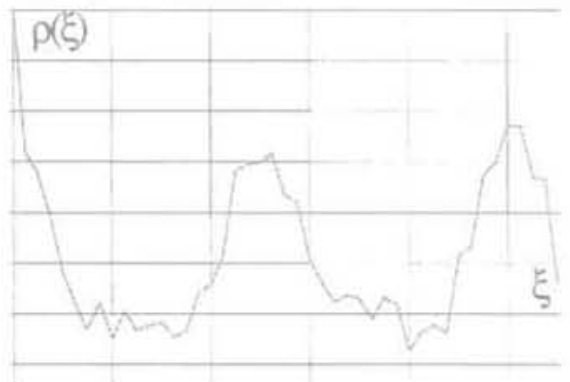

(b) Auto-corrélogramme

FG. 7 Auto-corrélation d'un massif stratifié idéalisé.

Autocorrelation of an idealised stratified soil mass. 

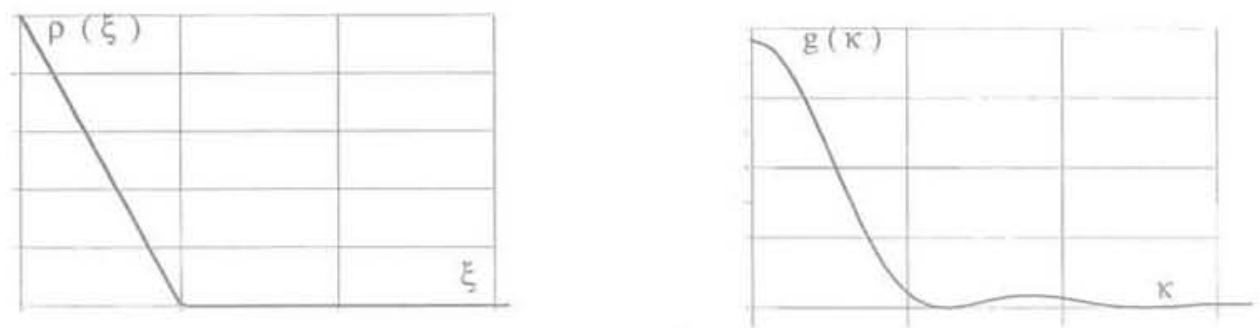

Décroissance linéaire
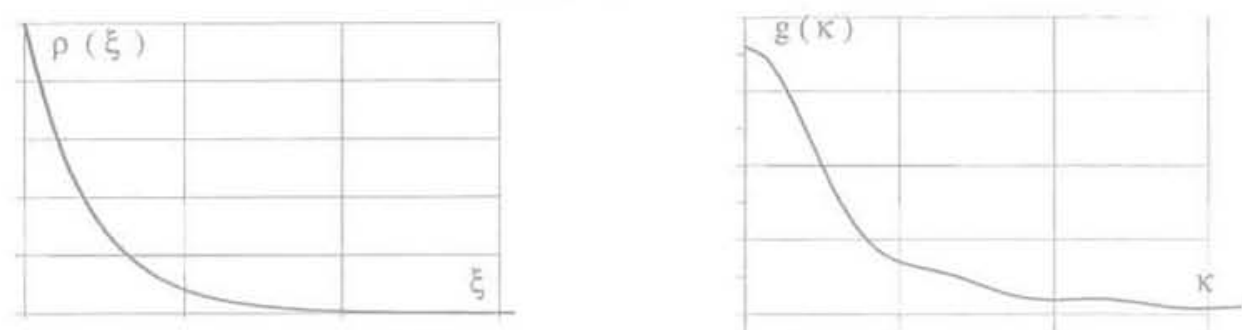

Décroissance exponentielle

FIG,8 Fonctions d'auto-corrélation (à gauche), et leur spectre de puissance (à droite). Autocorrelation functions (left) and their power spectra (right).

\section{Modèles d'auto-corrélation avec oscillations}

Un des rares modèles de ce type a été proposé par Baker et Zeitoun (1987), en utilisant le Principe d'entropie (de l'information) maximum. Ils ont appliqué simplement un filtre " passe bas ») à un bruit blanc gaussien. En d'autres termes, ils ont supposé que les variations des propriétés du milieu étaient purement erratiques, en limitant leur portée à une longueur minimum que l'on pourrait mettre en relation avec la dimension du volume d'échantillonnage de la grandeur mesurée.

Ce modèle extrêmement simple, à un seul paramètre, donne un résultat assez proche des auto-corré- logrammes expérimentaux, avec des incursions de $p$ dans la partie négative (Fig. 9).

L'auto-corrélogramme du massif stratifié idéalisé présenté sur la figure 7 peut aussi être transformé en spectre de puissance (Fig. 10).

On remarque ici que le maximum du spectre de puissance ne se présente plus à l'origine, mais en une valeur de $\kappa$ qui correspond à la principale longueur d'onde des oscillations de $\rho$, c'est-à-dire au « pas» des sous-couches du massif. On peut encore noter que, en toute rigueur, le spectre de puissance devrait rester strictement non-négatif, mais les petites excursions dans la partie négative résultent des approximations numériques lors des transformations.
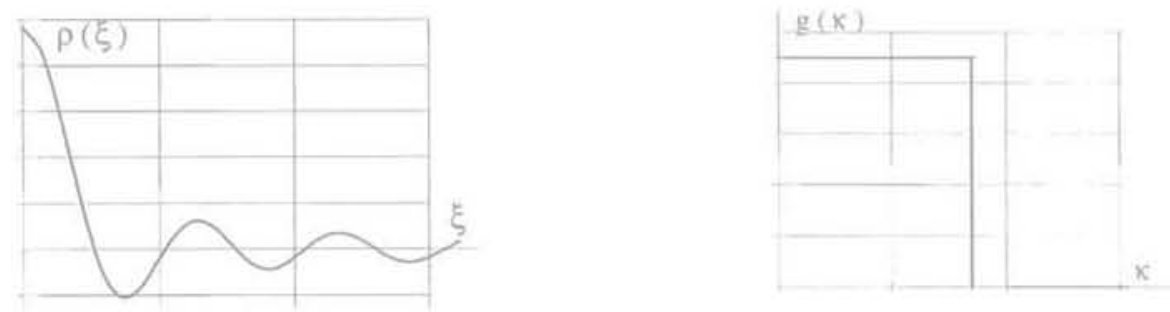

FG.9 Fonction d'auto-corrélation (à gauche) d'un bruit blanc gaussien filtré (à droite). Autocorrelation function (left) of a filtered gaussian white noise (right)
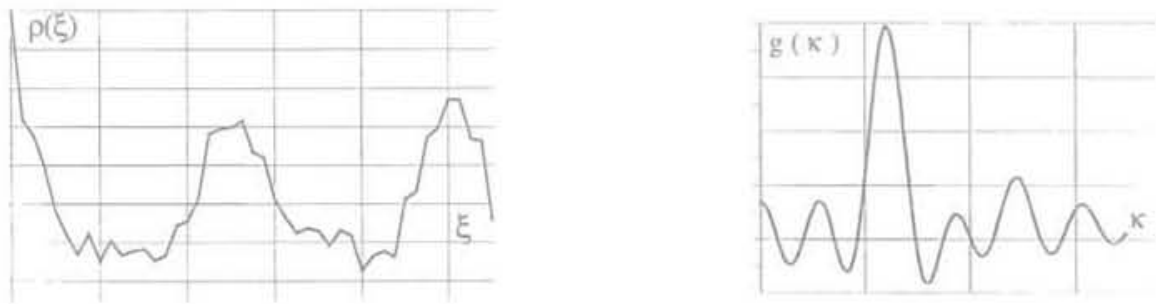

FIG.10 Auto-corrélation d'un massif stratifié (à gauche) et spectre correspondant (à droite). Autocorrelation function of a stratified soil mass (left) and associated spectrum (right). 


\section{Construction d'un modèle d'auto-corrélation}

\section{Proposition pour un modèle d'auto-corrélation}

À l'exception du modèle de Baker et Zeitoun (1987), les modèles à un seul paramètre semblent peu aptes à décrire correctement la structure d'auto-corrélation des propriétés observées dans les sols et les massifs rocheux. Un second paramètre devra donc être introduit.

La construction est réalisée en choisissant un spectre de puissance, puis en le transformant en fonction d'auto-corrélation.

Plusieurs modèles pourraient correspondre aux principes évoqués plus haut. A titre d'exemple, on peut choisir un spectre de puissance en forme d'exponentielle décroissante caractérisée par un paramètre $\lambda$, bornée à gauche par une longueur d'onde $L$ :

$$
g(\kappa)=S \cdot \exp \left(-\frac{\kappa}{\lambda}\right) \quad \forall \kappa \geq \frac{2 \cdot \pi}{L}
$$

Ce spectre correspond à une fonction d'auto-corrélation avec oscillations :

$$
\rho(\xi)=\frac{1}{1+(\xi \cdot \lambda)^{2}} \cdot\left(\cos \frac{2 \cdot \pi \cdot \xi}{L}-\xi \cdot \lambda \cdot \sin \frac{2 \cdot \pi \cdot \xi}{L}\right)
$$

Cette fonction d'auto-corrélation et le spectre correspondant sont représentés côte à côte sur la figure 11.

\section{2}

\section{Ajustement sur des données expérimentales}

Les paramètres $L$ et $\lambda$ peuvent étre obtenus par ajustement sur les auto-corrélogrammes expérimentaux $\rho(\xi)$, en utilisant la méthode des moindres carrés.

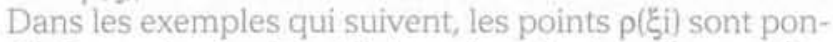
dérés par le nombre $N^{\prime}$ de paires de données expérimentales, comme défini par la relation (1).
La figure 12 montre les résultats de l'ajustement sur les deux exemples de résultats expérimentaux déjà présentés sur les figures 3 et 4 . On peut y remarquer un certain nombre de caractéristiques qui ne sont pas de simples coïncidences.

Pour les essais au pénétromètre, le paramètre $L$ peut être mis en relation avec une alternance assez régulière d'autres propriétés, d'un pas d'environ $1,50 \mathrm{~m}$, qui avait été clairement identifiée par des analyses stratigraphiques. Dans les deux exemples, le second paramètre pourrait correspondre à une dimension caractéristique du volume d'échantillonnage.

Enfin, pour le cas des modules pressiométriques du massif rocheux, le paramètre $L=25,6$ m correspond à un phénomène principal, de grande longueur d'onde. On pourrait aisément y superposer un second phénomène de plus courte longueur d'onde ( 4 à 5 mètres).

Ce travail de développement d'un modèle reste à améliorer, mais on peut déjà constater sur ces deux exemples une concordance très satisfaisante avec les observations expérimentales. La forme du spectre pourrait être remplacée par une courbe plus douce, mais toujours caractérisée par deux paramètres équivalents : (1) une valeur centrale, ou un pic, et (2) une dispersion autour de ce pic, ce qui conciuirait encore à des auto-corrélogrammes avec oscillations amorties.

\section{3}

\section{Application à deux dimensions}

La notion d'auto-corrélation peut être définie, comme dans l'équation (1), pour une distance $\xi$ mesurée selon une seule direction (1D), généralement la profondeur, pour une direction quelconque (isotrope), ou pour 2, voire 3 directions (2D et 3D).

Dans la pratique, le nombre de mesures disponibles limite souvent les approches à 2 ou 3 dimensions. Dans ce cas, pour obtenir un nombre significatif de paires de points de mesure pour une direction donnée, on est amené à relâcher un peu les conditions, par exemple en remplaçant les points de mesure par un volume de mesure centré sur le point.

Les résultats correspondant à 191 modules pressiométriques mesurés dans un massif rocheux très hétérogène ont déjà été présentés sur la figure 4 sous la forme d'un auto-corrélogramme isotrope. Les mêmes données ont aussi été analysées en 2 dimensions dans le plan horizontal. Chaque auto-corrélogramme expérimental, correspondant à une direction donnée, a été ajusté sur le modèle en exponentielle tronquée, donnant ainsi une paire de valeurs $L$ et $1 / \lambda$ pour chaque direction. Ces deux paramètres sont reportés, en coor-
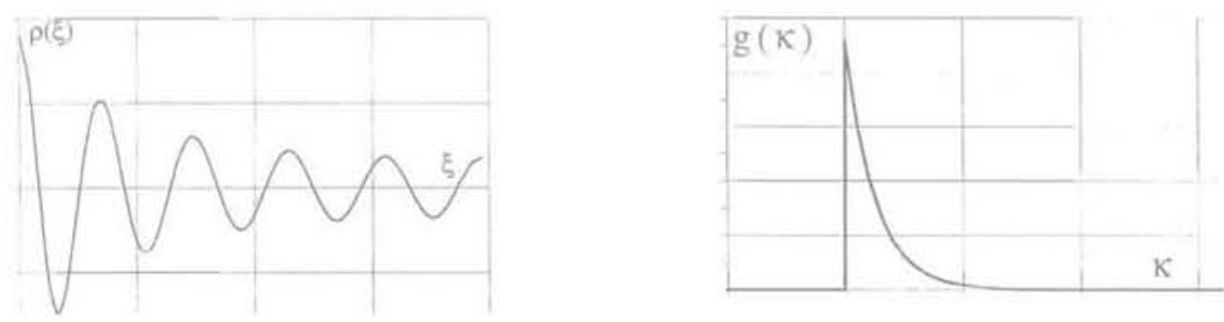

FIG. 11 Fonction d'auto-corrélation (à gauche) obtenue par la transformation d'un spectre en exponentielle tronquée (à droite). Autocorrelation function (left) obtained from the truncated exponential spectrum (right). 


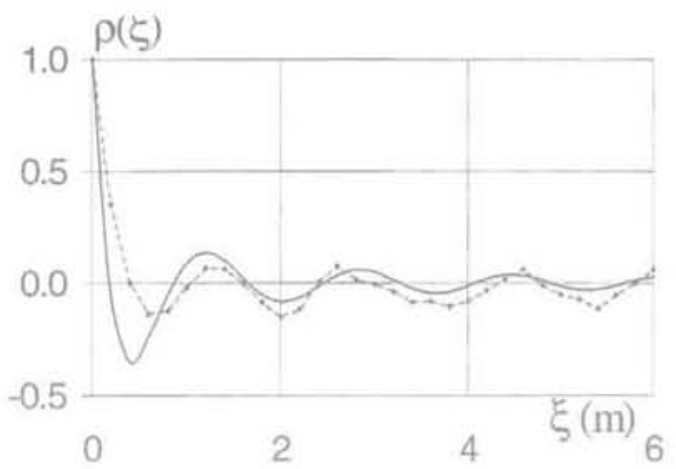

(a) Pénétromètres $C P T(b)$

$\mathrm{L}=1,63 \mathrm{~m}$ et $\mathrm{I} / \mathrm{\lambda}=0,17 \mathrm{~m}$

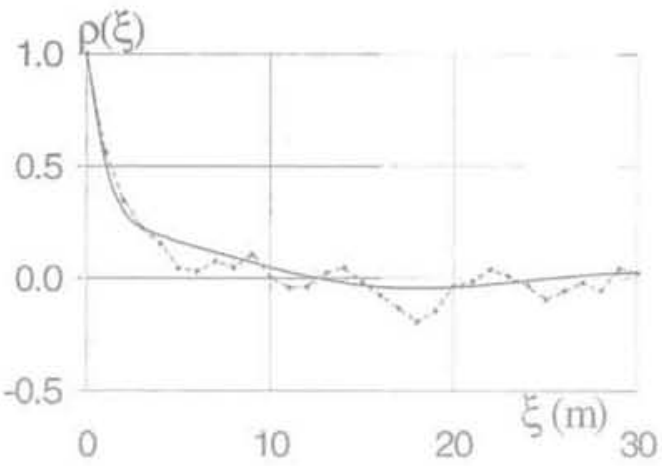

(b) Modules pressiométriques $\mathrm{L}=25.6 \mathrm{~m}$ et $1 / \lambda=-0.84 \mathrm{~m}$

FIG. 12 Exemples d'ajustement sur des auto-corrélogrammes expérimentaux.

Examples of fitting to experimental autocorrelograms.

données polaires, sur la figure 13, manifestant clairement une allure elliptique.

Une ellipse a ensuite pu être ajustée sur ces points, caractérisée par trois paramètres : la longueur des deux axes et un angle $\alpha$ donnant la direction du grand axe par rapport au nord:

$$
\begin{aligned}
& \left(\frac{r \cdot \cos (\theta+\alpha)}{L_{1}}\right)^{2}+\left(\frac{r \cdot \sin (\theta+\alpha)}{L_{2}}\right)^{2}=1 \\
& \left(\lambda_{1} \cdot r \cdot \cos (\theta+\alpha)\right)^{2}+\left(\lambda_{2} \cdot r \cdot \cos (\theta+\alpha)\right)^{2}=1
\end{aligned}
$$

Les orientations des axes principaux des deux courbes coïncident pratiquement, de mème que l'allongement relatif des ellipses (rapport de grand axe au petit axe). De toute évidence, ces « directions principales " traduisent une certaine structure interne, cachée, du massif rocheux considéré, inaccessible à une analyse classique, mais apparaissant clairement au travers de la procédure proposée.

\section{6}

\section{Conclusions relatives à l'étude de la variabilité spatiale}

Les modèles classiques d'auto-corrélation, appliqués aux propriétés des sols et des massifs rocheux, ne s'adressent qu'à la première partie de la courbe, correspondant à la " persistance ») ou la " portée ) des propriétés. Mais les auto-corrélogrammes expérimentaux habituellement observés comportent toujours, après la décroissance monotone proche de l'origine, des oscillations plus ou moins régulières autour de zéro, qui résultent du caractère périodique lié à l'alternance des propriétés.

Le modèle proposé s'ajuste de manière assez satisfaisante aux données expérimentales. Ce modèle, qui pourrait encore subir diverses améliorations, utilise seulement deux paramètres : (1) une longueur d'onde principale et (2) une " distance d'amortissement v, ces paramètres ayant tous deux la dimension d'une longueur.

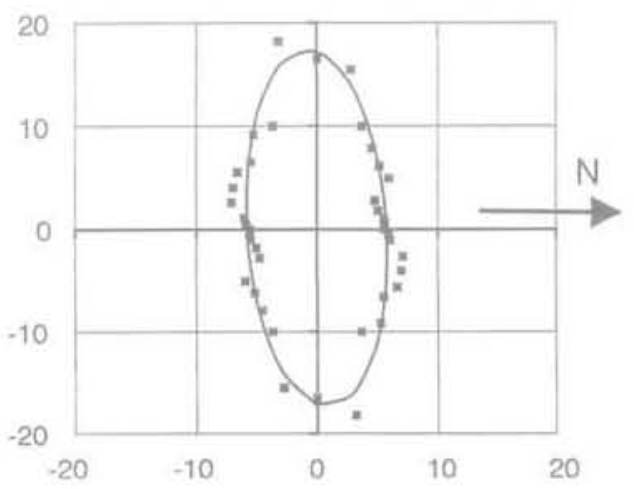

(a) Paramètre $L(m)$ $\mathrm{L}_{1}=17,4, \mathrm{~L}_{2}=5,8, \alpha=-87^{\circ}$

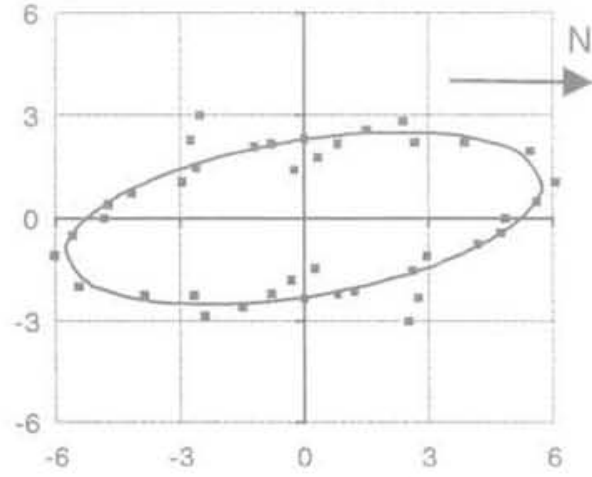

(b) Paramètre $1 / \lambda(m)$

$1 / \lambda_{1}=-5,8,1 / \lambda_{2}=-2,3, \alpha=12^{\circ}$

AG.13 Paramètres des auto-corrélogrammes 2D : valeurs expérimentales et ellipses ajustées. Parameters of 2D autocorrelograms : experimental data and fitted ellipses. 
Les analyses peuvent être menées à une seule dimension : la verticale pour les couches sédimentaires ou une direction quelconque (isotrope), pour les massifs rocheux à structure complexe indéterminée. Elles peuvent aussi, lorsque le volume d'information disponible est suffisant, être menées à deux ou à trois dimensions, permettant ainsi de mettre en évidence l'anisotropie de la structure d'auto-corrélation. Les paramètres peuvent alors apparaître sous la forme d'ellipses ou d'ellipsoïdes de paramètres principaux, avec une signification proche des notions de contraintes ou déformations principales connues en théorie de l'élasticité.

\section{7}

\section{Prise en compte de la variabilité spatiale dans les applications}

\section{Position du problème}

Dans une couche de sol (ou un massif rocheux) homogène " en grand $n$, une propriété géotechnique mesurée en différents points pourra être assimilée à un champ aléatoire, et représentée par les composantes suivantes :

- la dérive qui est une fonction décrivant l'évolution, sur le domaine considéré, des moyennes et variances locales, c'est-à-dire estimées sur un petit sousdomaine; cette fonction comportera éventuellement une transformation de la variable de base ; elle prendra une forme simple adaptée à chaque cas particulier (linéaire, quadratique ou autre) :

- après déduction de cette fonction, les données expérimentales forment un champ aléatoire homogène (jusqu'au second moment statistique), ou stationnaire : la moyenne est souvent choisie égale à zéro au cours de l'étape (1), et la variance, rendue constante sur le domaine, reste le paramètre unique ;

- la structure de la variabilité spatiale résiduelle est représentée par la fonction d'auto-corrélation, à une ou plusieurs dimensions, qui peut être décrite par un ou deux paramètres; le caractère oscillant de celte fonction constitue une caractéristique fondamentale observée sur les matériaux naturels.

La prise en compte d'une telle propriété géotechnique dans une application requiert la simulation de la valeur prise en tout point par cette propriété. Mais, les données expérimentales disponibles sont toujours trop peu nombreuses, et il faut donc "reconstruire » ces valeurs sur le domaine. Des techniques optimisées d'interpolation existent, comme le krigeage (Krige, 1962). Ces techniques peuvent fournir en tout point une estimation de la grandeur, sous la forme d'une valeur probable et de l'incertitude qui y est attachée.

La simulation par la méthode des champs aléatoires permet de conserver le caractère incertain, aléatoire, des valeurs possibles de la grandeur en tout point du domaine étudié, Ces valeurs sont reconstruites comme la somme de deux composantes distinctes:

- une fonction déterministe décrivant la variation, sur le domaine considéré, de la moyenne (et éventuellement de la variance) ;

- une variation aléatoire de la propriété autour de cette moyenne, présentant une certaine amplitude, la variance, et une structure d'auto-corrélation données.

Cette seconde composante, de nature aléatoire, incertaine, sera introduite dans les applications et elle produira en finale une incertitude sur les résultats obtenus.

Il faut souligner que la simulation de la variabilité spatiale, comme proposée ci-dessus, entraîne systématiquement l'utilisation d'un grand nombre de variables aléatoires, qui sont simplement les valeurs, affectées d'incertitude, de la grandeur considérée en tous les points (ou volumes élémentaires) choisis pour représenter le domaine.

\section{2}

\section{Techniques de simulation existantes}

Plusieurs méthodes stochastiques, c'est-à-dire utilisant le hasard, ont été proposées pour simuler ces variations spatiales, par exemple pour fournir les données à introduire dans des calculs par éléments finis. Par exemple, Zeldin et Spanos (1995) améliorent les approches spectrales ou ARMA par le développement des ondelettes (wavelets).

La simulation pure du hasard, connue sous le nom de méthode de Monte-Carlo, reste la méthode de référence, mais le volume de calcul nécessaire pour réellement converger vers la solution peut atteindre une taille astronomique.

Des méthodes semi-analytiques, de premier ou de second ordre, ont aussi été développées, comme les éléments finis stochastiques (Cambou, 1975, Vanmarcke et al., 1986), en se basant sur des développements en séries de Taylor. Mais ces méthodes présentent diverses limitations et elles nécessitent la recherche (souvent réalisée par voie numérique) des dérivées partielles par rapport à chaque variable aléatoire.

Enfin, plusieurs méthodes d'estimation ponctuelle ont été proposées, inspirées de la technique imaginée par Rosenblueth (1975), mais elles sont très rapidement limitées (Bolle, 1988).

\section{3}

\section{Technique de simulation proposée}

Pour faire face aux difficultés et aux limitations inhérentes à ces méthodes de simulation, une technique de calcul originale a été mise au point (Bolle, 1988, 1994) pour simuler divers processus stochastiques avec de grands nombres de variables aléatoires. Cette méthode est plus performante que la simulation de Monte-Carlo, car le nombre de calculs nécessaires est très fortement réduit. Elle ne peut toutefois s'appliquer, en toute rigueur, qu'à des fonctions « gentiment » continues.

La méthode proposée utilise uniquement, de manière interne, des variables aléatoires non corrélées à distribution gaussienne. Dès lors, la modélisation des variables spatiales devra subir une transformation permettant de l'exprimer sous la forme d'un ensemble de 
variables aléatoires indépendantes (au sens de non corrélées) à distribution symétrique. Cette seconde condition est en général automatiquement respectée si l'analyse de la variabilité spatiale est limitée aux moments statistiques de second ordre.

\section{8}

\section{Méthode de calcul probabiliste proposée}

L'idée de base consiste à rechercher des estimateurs des premiers moments statistiques de la fonction étudiée, comme dans les méthodes basées sur le développement en séries de Taylor. Ces moments statistiques sont estimés par l'addition des effets dispersants, des incertitudes, attribuables à la variabilité de chaque variable aléatoire indépendante.

Plus précisément, les premiers moments statistiques de la distribution sont estimés en ajoutant à un terme central la somme des termes perturbateurs liés à chaque variable aléatoire indépendante. Le terme central représente la valeur non aléatoire de la fonction, c'est-à-dire la valeur obtenue lorsque toutes les variables sont considérées comme déterministes, ou de variance nulle.

Le calcul des perturbations, définies comme les effets générés par la dispersion de chaque variable, utilise une variante optimisée de la méthode des estimations ponctuelles de Rosenblueth (1975), avec un schéma de discrétisation plus élaboré, comportant trois points au lieu de deux. Une distribution continue est ainsi remplacée par une distribution discrète en respectant jusqu'au moment statistique de quatrième ordre inclus.

\section{1}

\section{Transformation du champ aléatoire en variables indépendantes}

En vue de la résolution d'un problème concret, les propriétés géotechniques connues sous la forme d'un champ aléatoire doivent être exprimées en une série de points (ou de volumes élémentaires) de l'espace. Un exemple habituel est un calcul par éléments finis, qui demande de connaitre les propriétés des matériaux en chaque élément, voire en chaque nceud.

On désignera par $X=\left(x_{1} x_{2} \ldots x_{N}\right)$ les valeurs de ces propriétés. Elles sont caracterisées par les moments statistiques d'ordre 1 et 2 de leur distribution couplée, soit :

- la moyenne des distributions $\mathrm{M}=\left(\begin{array}{lll}\bar{x}_{1} & \ldots \bar{x}_{N}\end{array}\right)$;

- les écarts types des distributions, donnés par la matrice diagonale $S=\left[\begin{array}{ccc}\sigma_{X_{1}} & & \varnothing \\ & \ddots & \\ \varnothing & & \sigma_{X_{N}}\end{array}\right]$;

- la matrice de corrélation $\mathbf{R}(\mathbf{X})$, qui comporte une diagonale unitaire et des termes symétriques exprimant l'auto-corrélation spatiale de la grandeur considérée; ces termes sont obtenus en calculant, avec le modèle proposé, la valeur de $\rho(\xi)$ pour chaque couple de points de l'espace.
Des variables réduites normées et centrées formant un vecteur $T$, parallèle à $X$, de moyenne nulle et de variance unitaire, sont définies par la transformation linéaire réversible :

$$
X=M+S \cdot T \Leftrightarrow T=S^{-1} \cdot(X-M)
$$

$\operatorname{avec} \mathrm{S}^{-1}=\left[\begin{array}{ccc}1 / \sigma_{X_{1}} & & \varnothing \\ \varnothing & & 1 / \sigma_{X_{N}}\end{array}\right]$

La matrice de corrélation de $\mathrm{R}(\mathrm{T}) \equiv \mathrm{R}(\mathrm{X})$ peut ètre transformée par une opération de diagonalisation, selon Rosenblatt (1952), en les éléments suivants : - une matrice diagonale formée des valeurs propres de $\mathrm{R}(\mathrm{T}) \equiv \mathrm{R}(\mathrm{X})$, que l'on peut regrouper sous la forme d'un vecteur $L=\operatorname{Diag}\left(\lambda_{1} \lambda_{2} \ldots \lambda_{\mathrm{v}}\right)$;

- la matrice (normée) A des vecteurs propres correspondant à ces valeurs propres.

On peut alors définir de nouvelles variables aléatoires notées $\mathrm{U}=\left(\mathrm{u}_{1} \mathrm{u}_{2} \ldots \mathrm{u}_{\mathrm{N}}\right)$ données par :

$$
\mathrm{U}=\mathrm{A} \cdot \mathrm{T}=\mathrm{A} \cdot \mathrm{S}^{-1}(\mathrm{X}-\mathrm{M})
$$

On peut montrer que ces nouvelles variables aléatoires sont indépendantes, de moyenne nulle, avec $\bar{U}=\left|\begin{array}{lll}0 & 0 \ldots 0\end{array}\right|$, et de variances $\sigma^{2}=L=\left|\lambda_{1} \lambda_{2} \ldots \lambda_{N}\right|$.

La transformation de $\mathbf{X}$, vecteur des variables réelles exprimées en unités physiques, en variables non corrélées $\mathrm{U}$, de moyenne nulle et de variances $\mathrm{L}$, est réversible:

$$
\mathrm{U}=\mathrm{A} \cdot \mathrm{S}^{-1} \cdot(\mathrm{X}-\mathrm{M}) \Leftrightarrow \mathrm{X}=\mathrm{M}+\mathrm{S} \cdot \mathrm{A}^{\mathrm{T}} \cdot \mathrm{U}
$$

en remarquant que, par suite des propriétés de $\mathrm{A}$, on a $\mathrm{A}^{-1}=\mathrm{A}^{\mathrm{T}}$.

On peut résumer ces transformations, en langage plus concret, en une mise à l'échelle des grandeurs physiques, puis en une rotation (dans un espace à $N$ dimensions) en tout point assimilable à la recherche des contraintes principales dans un corps élastique.

Bien entendu, il est possible d'inclure dans cette transformation toute autre variable aléatoire intervenant dans le problème, à condition de lui donner une distribution proche de la normale, et d'exprimer son éventuelle corrélation avec les autres variables.

\section{2}

\section{Estimation ponctuelle à trois points}

Chacune des variables aléatoires indépendantes $\mathrm{U}_{\mathrm{p}}$ à distribution normale de movenne nulle et de variance $\lambda$, peut ètre représentée de manière équivalente (jusqư'au quatrième moment statistique inclus) par la distribution discrète en trois points représentée sur la figure 14. Les caractéristiques de cette distribution discrète s'écrivent simplement:

$$
u_{1}=\sqrt{3 \lambda_{i}} \quad P_{1}=\frac{1}{6} \quad P_{0}=\frac{2}{3}
$$



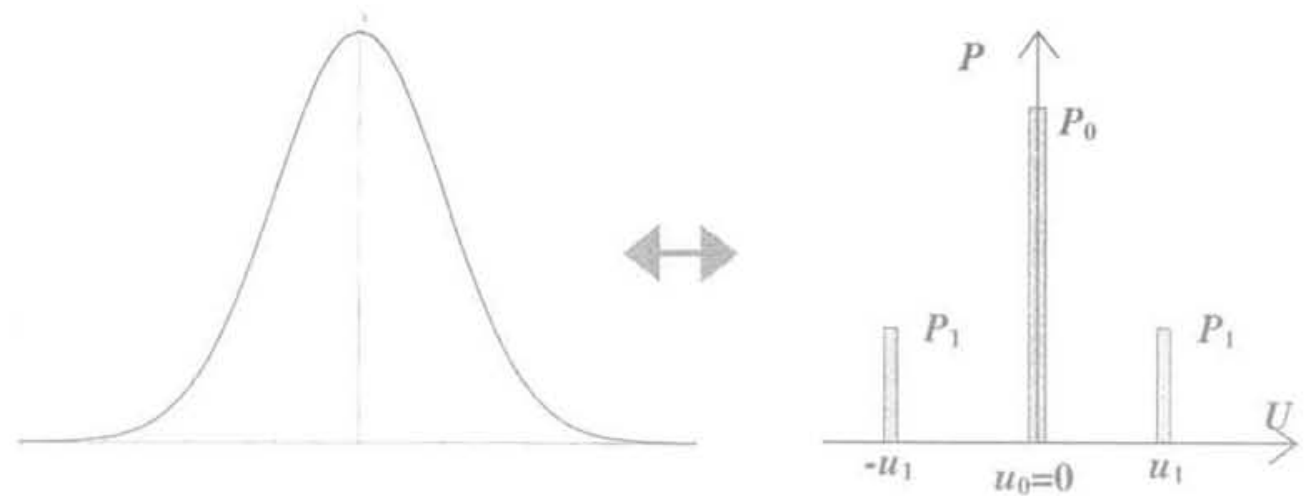

\section{3}

\section{Développement de la méthode de simulation}

La fonction $Y(X)$ représente le phénomène à simuler en faisant appel, par exemple, à un code de calcul par éléments finis. Le résultat du calcul sera la distribution de $Y(X)$ exprimée sous la forme des premiers moments statistiques.

Le terme central $Y$, de la distribution de $Y(X)$ est la valeur de cette fonction calculée au point central (moyenne) de chaque variable aléatoire $\mathrm{X}$ :

$$
Y_{s}=Y(\bar{X})=Y(M)
$$

Il s'agit du moment statistique d'ordre 1, non perturbé, de la fonction $\mathrm{Y}(\mathrm{X})$. De même, la valeur non perturbée des moments statistiques d'ordre supérieur vaudra respectivement :

- variance (ordre 2 ) =0 ;

- asymétrie (ordre 3 ) =0;

- aplatissement (ordre 4) =3, c'est-à-dire la valeur correspondant à une distribution normale.

Les perturbations sont estimées par rapport à ces tendances centrales (valeurs non perturbées), à l'aide des valeurs de la fonction $\mathrm{Y}(\mathrm{X})$ calculées aux deux points latéraux de la discrétisation de la distribution de chaque variable aléatoire, que l'on appellera les valeurs perturbées de la fonction. On désigne les valeurs de la fonction $\mathrm{Y}(\mathrm{X})$ calculées en ces deux points, pour la variable $\mathrm{U}$ d'indice $\mathrm{i}$, par la notation :

$$
\left\{\begin{array}{l}
Y_{i}^{*}=Y\left(M+S \cdot A^{\top} \cdot\left(0, \ldots,+u_{j}, \ldots, 0\right)\right) \\
Y_{F}=Y\left(M+S \cdot A^{\top} \cdot\left(0, \ldots,-u_{i}, \ldots, 0\right)\right)
\end{array}\right.
$$

La distribution discrète de la fonction $\mathrm{Y}(\mathrm{X})$, dans laquelle une seule variable $U$ d'indice $\mathrm{i}$ est considérée comme aléatoire, se présente alors sous la forme représentée sur la figure 15.

Les notations suivantes simplifieront les écritures:

$$
\delta_{i}^{+}=Y_{i}^{+}-y_{i}=\frac{1}{6} \cdot\left(5 \cdot d_{i}^{+}-d_{i}^{-}\right)_{\text {et }}
$$

$$
\begin{gathered}
\delta_{i}^{-}=Y_{i}^{-}-y_{1}=\frac{1}{6} \cdot\left(-d_{i}^{+}+5 d_{i}^{-}\right) \\
\varepsilon_{i}=y_{i}-Y_{c}=\frac{1}{6} \cdot\left(d_{i}^{+}+d_{i}^{-}\right) \\
\eta_{i}=\frac{d_{i}^{+} \cdot d_{i}^{-}}{36}
\end{gathered}
$$

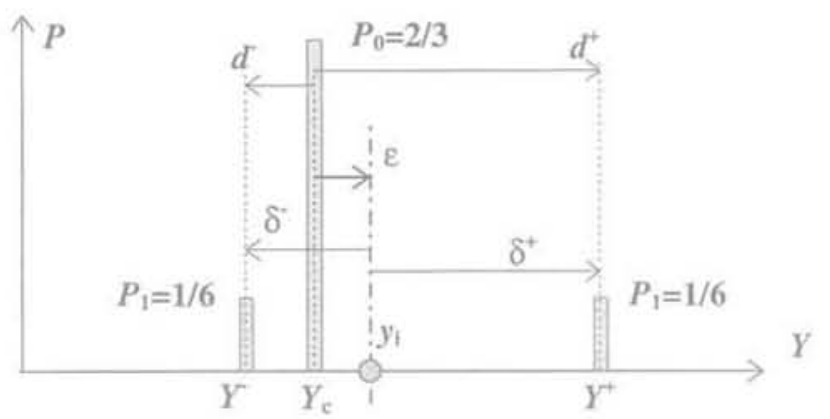

FIG is Calcul des termes perturbateurs de $\mathrm{Y}(\mathrm{X})$ sous l'effet de la variance de Ui.

Calculation of the perturbing terms of $Y(X)$ due to the variance of $U_{1}$.

L'estimateur de la moyenne de $Y(\mathrm{X})$, soit $\mathrm{y}_{1}$, peut alors être aisément exprimé par :

$$
y_{i} \cong Y_{c}+\varepsilon_{i}
$$

Pour les moments d'ordre supérieur à un, on adoptera comme principe que la seule variable U d'indice i est aléatoire et, par conséquent, que la distribution discrète de la fonction $\mathrm{Y}(\mathrm{X})$ est complètement représentée par ce schéma en trois points. Moyennant cette hypothèse, on considère que $y_{i}$ est une moyenne locale de la distribution, et que les $\delta^{2}$ sont des perturbations locales nettes de cette moyenne.

Les moments perturbateurs d'ordre k seront obtenus par la formule générale suivante:

$$
\begin{aligned}
& d \mu_{k, f} \equiv \sum_{j} P_{i} \cdot\left(y-y_{i}\right)^{k} \\
& =P_{0} \cdot\left(-\varepsilon_{i}\right)^{k}+P_{1} \cdot\left(\left(\delta_{i}^{+}\right)^{k}+\left(\delta_{i}^{-}\right)^{k}\right)
\end{aligned}
$$


à partir de laquelle on peut obtenir les diverses expressions correspondant à la variance, la dissymétrie et l'aplatissement.

Ensuite, compte tenu de l'indépendance des variables de type $\mathrm{U}$, on pose que la perturbation totale des différents moments statistiques de $Y(\mathrm{X})$ peut être obtenue par une simple sommation des effets des perturbations dues à chaque variable.

L'estimation de la moyenne de la distribution de la fonction $Y(\mathrm{X})$ sera ainsi donnée par :

$$
\bar{Y} \equiv Y_{s}+\frac{1}{6} \sum_{i=1}^{N}\left(d_{i}^{+}+d_{i}^{-}\right)=Y_{c}+\sum_{i=1}^{N} \varepsilon_{i}
$$

La variance de la distribution de la fonction $Y(X)$ sera estimée par une expression similaire :

$$
\sigma^{2}(Y) \equiv \sum_{i=1}^{N} d \mu_{2, i}=\sum_{i=1}^{N}\left(5 . \varepsilon_{j}^{2}-12 . \eta_{i}\right)
$$

La dissymétrie et l'aplatissement de la fonction $Y(X)$ peuvent être estimés par des développements conduisant à des expressions similaires.

\section{4}

\section{Calcul simultané de plusieurs fonctions et estimation des corrélations}

Dans la première partie des développements, pour ne pas alourdir l'écriture, on n'a considéré qu'une seule fonction scalaire $Y(X)$ sans indice. Mais on a plus souvent affaire à plusieurs fonctions simultanées $Y(X)=\left\{Y_{1}(X), Y_{2}(X) \ldots, Y_{M}(X)\right\}$. Il suffit de penser, par exemple, aux résultats fournis par la méthode des éléments finis, qui sont obtenus à chaque phase de calcul en tous les nœuds (ou points d'intégration) du réseau.

Les moments statistiques croisés d'ordre deux (covariances) de tous les couples de fonctions $\left\{Y_{i}(\mathrm{X}), Y_{k}(\mathrm{X})\right\}$ peuvent aussi être calculés par la même méthode. Pour chacune de ces fonctions, on peut encore calculer le terme central de leur distribution, $Y_{\mathrm{j}, \mathrm{c}}$ et $Y_{\mathrm{k}, \mathrm{c}^{\prime}}$ qui sont la valeur de la fonction calculée au point central de chaque variable aléatoire. Il s'agit des moyennes non perturbées des fonctions:

$$
Y_{i c}=Y_{j}(\bar{X})=Y_{i}(\mathbf{M}) \text { et } Y_{k i c}=Y_{k}(\bar{X})=Y_{k}(\mathbf{M})
$$

On désignera les valeurs perturbées des fonctions $Y_{\mathrm{j} . \mathrm{e}}$ et $Y_{\mathrm{k} . \mathrm{c}}$ calculées aux deux points latéraux de la discrétisation de la distribution de la variable $\mathrm{U}$ d'indice $i$ par la notation:

$$
\left\{\begin{array}{l}
Y_{j, i}^{ \pm}=Y_{j}\left(\mathbf{M}+\mathbf{S} \cdot \mathbf{A}^{\mathrm{T}} \cdot\left\{0, \ldots, \pm u_{j}, \ldots, 0\right\}\right) \\
Y_{k, j}^{ \pm}=Y_{k}\left(\mathbf{M}+\mathbf{S} \cdot \mathbf{A}^{\mathrm{T}} \cdot\left\{0, \ldots, \pm u_{j}, \ldots, 0\right\}\right)
\end{array}\right.
$$

Par des développements similaires, qui ne seront pas exposés ici, on peut ensuite obtenir une estimation de la covariance, puis de la corrélation entre chaque couple de variables résultats $Y_{j}(\mathrm{X})$ et $Y_{k}(\mathrm{X})$ :

$$
\begin{aligned}
& \operatorname{cov}\left(Y_{j}, Y_{k}\right) \equiv \sum_{i=1}^{N} d \mu_{j k, i} \\
& =\sum_{j=1}^{N}\left(\frac{d_{j, i}^{+} \cdot d_{k, i}^{+}-d_{j, i}^{-} \cdot d_{k, i}^{-}}{6}-\varepsilon_{j, i}, \varepsilon_{k, i}\right)
\end{aligned}
$$

$$
\rho\left(Y_{j}, Y_{k}\right)=\frac{\operatorname{cov}\left(Y_{j}, Y_{k}\right)}{\sigma\left(Y_{j}\right) \cdot \sigma\left(Y_{k}\right)}
$$

Dans la conduite pratique des calculs, il suffit d'ajouter aux opérations relatives à chacune des fonctions $Y(X)$ considérées isolément, une sommation relative à chaque couple $(j, k)$.

\section{Remarques sur les volumes de calcul}

L'amélioration apportée par une telle méthode par comparaison avec les autres méthodes de simulation réside d'abord dans le nombre de simulations de $Y(X)$, qui est réduit à $2 . N+1$ pour $N$ variables aléatoires. Ceci permet l'approche de problèmes faisant intervenir de grands nombres de variables aléatoires. On peut remarquer que ce nombre de simulations correspond précisément au nombre idéal permettant une utilisation optimale de l'information de départ.

Dans un problème à $N$ variables aléatoires tel que nous l'avons défini, on dispose de $2 . N$ données qui sont les deux premiers moments statistiques de chaque variable. L'indépendance entre ces variables est assurée par la technique d'estimation elle-même, qui utilise des variables aléatoires rendues non corrélées. Au calcul déterministe habituel, on ajoute donc strictement le nombre de simulations qui correspond au nombre d'informations élémentaires présentes dans les données.

On peut considérer qu'il n'y a de la sorte aucune perte ou dilution d'information dans le processus ou, en d'autres termes, aucune augmentation de l'entropie de l'information.

De surcroit, le nombre de simulations nécessaires, estimé a priori à $2 . N+1$, peut souvent être fortement réduit. En effet, lors de la transformation des variables $\mathrm{X}$ en variables non corrélées de type $\mathrm{U}$, les valeurs propres sont obtenues en ordre décroissant, et elles deviennent souvent négligeables au-delà d'un certain rang, soit $n<N$. Or, ces valeurs propres représentent la variance des variables de type U. Si cette variance devient négligeable, le caractère aléatoire des variables correspondantes disparaît, et elles se réduisent à des grandeurs déterministes, fixes, égales à zéro.

Dans l'application de la méthode, les variables $U$ d'indice $i>n$ produiront des perturbations nulles ou négligeables. Les simulations correspondantes sont inutiles, et le nombre total de simulations nécessaires est ramené à $2 . n+1$. Dans les cas habituels, $n$ dépasse rarement quelques dizaines. 


\section{Justification et domaine d'application de la méthode}

La justification de base de la méthode se fonde sur l'hypothèse que les effets du caractère aléatoire de chaque variable aléatoire indépendante sont aussi indépendants, et qu'ils peuvent dès lors être additionnés. La justification de cette hypothèse, et en particulier l'additivité des effets de chaque variable aléatoire indépendante, n'a toutefois pas encore été étudiée in extenso par des principes mathématiques rigoureux.

Mais des considérations sur la bonne utilisation de l'information, basées sur le concept d'entropie de l'information, permettent intuitivement de fournir une certaine justification logique au choix de cette technique.

Enfin, de nombreuses applications ont été réalisées sur des fonctions simples, mais fortement non linéaires, et comparées de manière très satisfaisante avec les solutions théoriques. Des cas pratiques plus complexes ont été comparés aux résultats fournis par d'autres méthodes de simulation, dont la méthode de référence de Monte-Carlo.

La seule réserve à l'application de la méthode concerne la continuité des fonctions analysées. Compte tenu de la manière de calculer les perturbations, qui fait intervenir un calcul des différentielles d'ordre 1 et 2, il faut que les dérivées estimées par ces différentielles existent sur le domaine balayé par la fonction.

Un contre-exemple de cette nature a été mis en évidence par Fischbach (1995) dans une application consacrée à la stabilité des pentes dans un milieu aléatoire stratifié. Au cours de la simulation, on a parfois constaté que la ligne de glissement (cercle) critique, qui passait généralement par le pied du talus, se trouvait assez brutalement " attirée ») par une couche anormalement moins résistante. Ce saut brutal n'est pas compatible avec les hypothèses de la méthode, et ce type de situation nécessite alors le recours à une technique plus générale comme Monte-Carlo.

\section{$\overline{\text { Bibliographie }}$}

Baker R., Zeitoun D.G. (1987) - « Soil variability and the maximum entropy principle n. ICASP 5, Vancouver, p. 642-649.

Bolle A. (1988) - « Approche probabiliste en mécanique des sols avec prise en compte de la variabilité spatiale o. Thèse $n^{\circ} 743$. EPF Lausanne.

Bolle A., Bonnechère F., Crémer J.-M. (1989) $-\pi$ Probabilistic approach of the settlement of an arch bridge $v$, XII ICSMFE, Rio de Janeiro.

Bolle A. (1990) - a Méthodes statistiques en infrastructures $\%$. Notes de cours à l'université de Liège (non publié).

Bolle A. (1994) - How to manage the spatial variability of natural soils. Probabilities and Materials. D. Breysse, ed. p. 505-516.

Cambou B. (1975) - « Application of firstorder uncertainty analysis in the finite element method in linear elasticity 7.
Proceedings, Second International Conference on Application of Statistics and Probability in Soil and Structure Engineering, Aachen, p. 67-87.

De Beer E., Lousberg E., Wallays M., Carpentier R.. De Jaeger J. Paquay J. (1977) - $\ll$ Bearing capacity of displacement piles in stiff fissured clays $\gg$. Verslag over navorsingen $n^{\circ} 39$ van het IWONL. Brussels.

Fischbach S. (1995) - "Etude de la stabilité des talus dans un cadre probabiliste ». Travail de diplôme, Université de Liège.

Krige D.G. (1962) - "Economic aspects of stoping through unpayable ore .. Journal South African Institute of Mining and Metallurgy.

Maertens J. (1990) - a Aanwending van de statistiek voor de verwerking van grondmechanische karakteristieken $»$.
Geoproba 90 (ed. Belgian Group of the ISSMFE), Brussels.

Matheron G. (1965) - Les variables régionalisées et leur estimation. Une application de la théorie des fonctions aléatoires aux sciences de la nature. Paris, Masson et Cie.

Rosenblatt M. (1952) - „ Remarks on a multivariate transformation 1. Ann. Math. Stat., vol. 13, p. 470-472.

Rosenblueth E. (1975) - « Point estimates for probability moments 1 . Proc. Nat. Acad. Sci. USA, vol. 72, n 10.

Vanmarcke E., Shinozuka M, Nagakiri S., Schuëller G.1. Grigoriu M. (1986) " Random fields and stochastic finite elements n. Structural Safety 3, p. 143166.

Zeldin B. Spanos P. (1995) - « Random field simulation using wavelet bases x. ICASP 7, Paris, p. 1275-1283. 